\title{
BRCA testing delay during the COVID-19 pandemic: How to act?
}

\author{
Angelo Minucci ${ }^{1}$ - Giovanni Scambia ${ }^{2,3} \cdot$ Maria De Bonis $^{1} \cdot$ Elisa De Paolis ${ }^{1} \cdot$ Concetta Santonocito ${ }^{1,3}$. \\ Anna Fagotti ${ }^{2,3} \cdot$ Ettore Capoluongo $^{4} \cdot$ Paola Concolino $^{1} \cdot$ Andrea Urbani $^{1,3}$
}

Received: 30 October 2020 / Accepted: 3 December 2020 / Published online: 12 December 2020

(C) The Author(s), under exclusive licence to Springer Nature B.V. part of Springer Nature 2020

\begin{abstract}
Recently, our lab, part of a referral center in Italy, reported its experience regarding the execution of germline BRCA1/2 $(g B R C A)$ testing during the first months of the coronavirus disease-2019 (COVID-19) pandemic, which highlights a substantial reduction (about $60 \%$ ) compared with the first 2 months of the current year. This evidence appeared to be a lockdown effect due to extraordinary restriction measures to slow down the spread of SARS-CoV-2. In this study, we aimed to evaluate the overall effects of the ongoing pandemic on $g B R C A$ testing in our institution and to understand how COVID-19 has influenced testing after the complete lockdown (March 8-May 5, 2020). Additionally, we compared this year's trend with trends of the last 3 years to better monitor $g B R C A$ testing progress. This detailed analysis highlights two important findings: (1) $g B R C A$ testing did not increase significantly after the lockdown period (May-October 2020) compared with the lockdown period (March-April 2020), emphasizing that even after the lockdown period testing remained low. (2) Comparing the total tests per year (January-October 2017, 2018, 2019, with 2020), the impact of COVID-19 on $g B R C A$ testing is apparent, with similarities of trends registered in 2017. These evidences reveal a $g B R C A$ testing delay for cancer patients and healthy patients at this moment, and the new era of $g B R C A$ testing in the management of ovarian, breast, pancreas and prostate cancer patients has been seriously questioned due to the COVID-19 pandemic. As consequence, we underline that measures to guarantee oncogenetic testing (e.g., $g B R C A$ testing) along with new diagnostic/clinic strategies are mandatory. For these reasons, several proposals are presented in this study.
\end{abstract}

Keywords BRCA genes - Germline BRCA testing - Tumor BRCA testing · Coronavirus disease 2019 - COVID-19 pandemic in Italy

$\begin{array}{ll}\text { Abbreviations } \\ \text { COVID-19 } & \text { Coronavirus disease-2019 } \\ \text { BRCA } & \text { BRCA1/2 } \\ \text { PARPi } & \text { Poly (ADP-ribose) polymerases inhibitor } \\ \text { gBRCA } & \text { Germline } B R C A \\ \text { tBRCA } & \text { Tissue } B R C A \\ \text { PV } & \text { Pathogenic variant }\end{array}$

Angelo Minucci

angelo.minucci@policlinicogemelli.it

1 Molecular and Genomic Diagnostics Unit, Fondazione Policlinico Universitario A. Gemelli IRCCS, Rome, Italy

2 Division of Oncological Gynecology, Department of Women's and Children's Health, Fondazione Policlinico Universitario A. Gemelli IRCCS, Rome, Italy

3 Università Cattolica del Sacro Cuore, Rome, Italy

4 Università Federico II-CEINGE, Biotecnologie Avanzate, Naples, Italy

\section{Introduction}

The novel coronavirus disease 2019 (COVID-19) pandemic is shattering healthcare systems worldwide. In addition to direct consequences of COVID-19 on patients' morbidity and mortality, secondary effects of the pandemic caused by national lockdown strategies on health and healthcare utilization are now apparent [1]. In fact, a great impact on cancer patient management is noticeable and many patients were unable to gain access to routine cancer screening and preventive services $[2,3]$.

Depending on cancer type and location, a delay of 6 weeks affects negatively in most cancers prognosis. The number of patients dying because of these delays could end up rivalling or exceeding COVID-19 deaths and several strategies have been proposed to ensure diagnosis, treatment and care for cancer patients during the ongoing pandemic [4]. Additionally to this potential crisis, increased burdens 
are placed on health systems and healthcare facilities, which impede timely and optimal care.

In this context, $B R C A 1 / 2$ (BRCA) genes can be considered an indicator, because gene testing has gained importance in ovarian, breast, pancreas and prostate cancer patient management for its usefulness in early diagnosis, prognosis, recurrent disease and therapy resistant tumor detection [5, 6]. Additionally, more recently, poly(ADP-ribose) polymerases inhibitors (PARPi) are playing an increasingly important role to treat $B R C A+$ cancer patients [7-10]. For this last reason, germinal $B R C A(g B R C A)$ testing, which became a fast track test, reached an important role as mandatory oncogenetic test to address patients towards targeted therapies.

In Italy, following the extraordinary restriction measures from March 8 to May 5, 2020, adopted by the government to encounter the spread of the COVID-19 pandemic, many genomic labs have been forced to re-modulated their priorities by temporarily interrupting most of their activities [11] or guaranteeing only those considered urgent and not postponable [12]. In this context, our lab, referral center for $g B R C A$ testing [13-15], recently documented changes regarding the execution of cancer testing in the midst of the pandemic [16]. A substantial reduction in $g B R C A$ testing, of about $60 \%$ compared with the first 2 months of the current year, was registered, but the requests have not been reset. Assuming that this drastic reduction in requests could be justified by the lockdown, imposed by the government, we aimed to evaluate the overall effects of the ongoing pandemic on $g B R C A$ testing in our institution and to understand how COVID-19 has influenced testing after the complete lock-down (March 8-May 5, 2020). Additionally, we compared this year's trend with trends of the last 3 years to better monitor $g B R C A$ testing progress.

\section{Methods}

We collected and analyzed data of a single center regarding the execution of the BRCA testing during the COVID-19 pandemic.

\section{Results}

Even after the lockdown, $g B R C A$ testing did not increase significantly compared with the lockdown months (March-April 2020), which underlines that during postlockdown overall $g B R C A$ testing did not significantly change and monthly numbers continue to be very different from that of the first months (January-February 2020) (Fig. 1).

As already highlighted in our previous paper, the trend of requests has been maintained with about $80 \%$ of testing coming from gynaecologists and oncologists; in conclusion, tests targeted ovarian, breast and pancreatic cancer patients. This evidence confirms a solid integration of $g B R C A$ testing in clinical oncology reinforcing the idea to use $g B R C A$ testing as an indicator for cancer patient management. However, the COVID-19 pandemic is limiting its use in clinical practice.

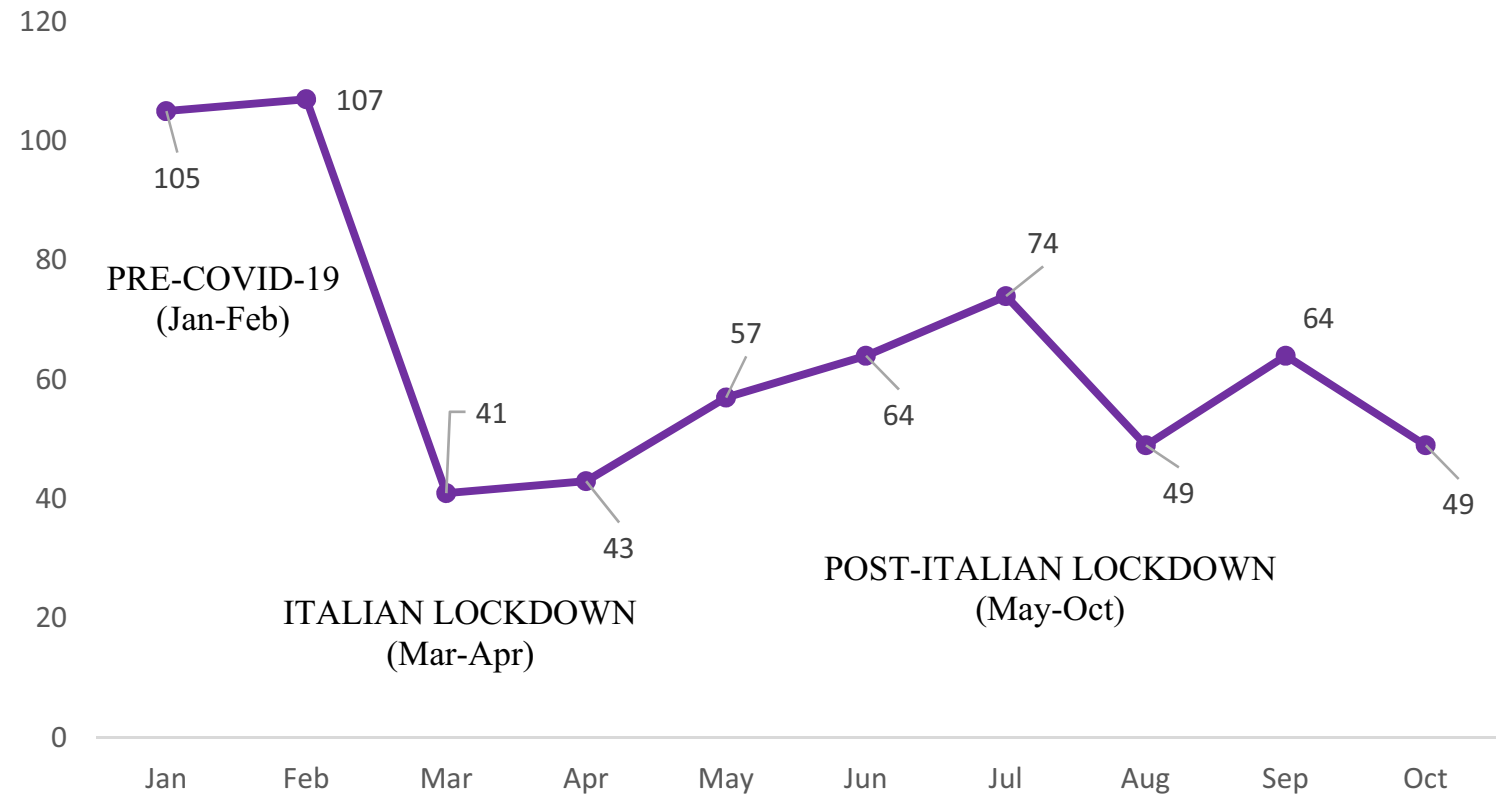

Fig. 1 Germline BRCA tests performed during the year 2020 (JanOct) in our lab. In lockdown months in Italy (Mar-Apr), there was a substantial reduction in the $g B R C A$ testing (about 60\%) compared to the first 2 months of the current year. However, even in the post lockdown months, tests/month continue to be significantly reduced compared to those of the pre-lockdown months 
In addition, to obtain more information on the trend of $g B R C A$ testing in the current year, we compared data of 2020 , with those of the last 3 years. During the lockdown months, tests were significantly lower than in all 3 years considered (2017: $-27 \%, 2018:-31 \%, 2019$ : -44\%) (Fig. 2). Even comparing the trend of the post-lockdown months, tests performed in 2020 are significantly reduced compared with 2019 and 2018 ( $-35 \%$ and $-32 \%$, respectively) and are only increased when compared with 2017 (+37\%) (Fig. 2). Moreover, comparing the overall tests per year (January-October) of the last 3 years, the impact of COVID-19 on $g B R C A$ testing is remarkable, shifting the trend of 2020 to that of 3 years ago.

At the same time, our lab performs tumor $B R C A$ $(t B R C A)$ testing from fresh frozen tissue for all patients with high-grade serous ovarian cancer for first diagnosis and for those patients with unresectable tumors undergoing biopsy. In this context, $t B R C A$ testing replaces $g B R C A$ testing, adding information by identifying those patients who are carriers of pathogenic variants (PVs) exclusively of the tumor [17]. It is interesting to note that $B R C A$ testing offered in the surgical setting, was not affected by the COVID-19 pandemic, e.g., overall $t B R C A$ testing compared with the last year resulted to be $349 t B R C A$ tests in 2019 versus 364 in 2020 (Fig. 3).

\section{Discussion}

Patients with breast and ovarian cancer have a high risk to harbor BRCA PVs. In addition, the risk of developing prostate cancer and pancreatic cancer increases in the presence of these PVs [6]. Knowledge regarding patients' predisposition to germline cancer enables the adoption of appropriate measures. Cancer prevention includes risk-reducing surgery and chemoprevention. Among the genes related to hereditary cancer syndrome, only BRCA genes have strong evidence of medical utility in preventive care.

COVID-19 profoundly affects healthcare systems worldwide and has the potential to affect negatively patients with diagnosed or newly diagnosed cancers. Time delays in treatment may result in cancer progression and/or more complicated treatment course. In fact, while certain tumors are indolent and slow growing, others are aggressive and need early treatment. A recent UK modelling study reports that treatment delays of 2-6 months leads to a substantial proportion of patients with early-stage tumor progression, changing the tumor from curable to incurable disease [18].

Our group recently reported a substantial reduction in $g B R C A$ testing (about $60 \%$ ) compared with the first 2 months of the current year in a referral center for $B R C A$ gene screening [16]. Starting from this evidence and assuming that this

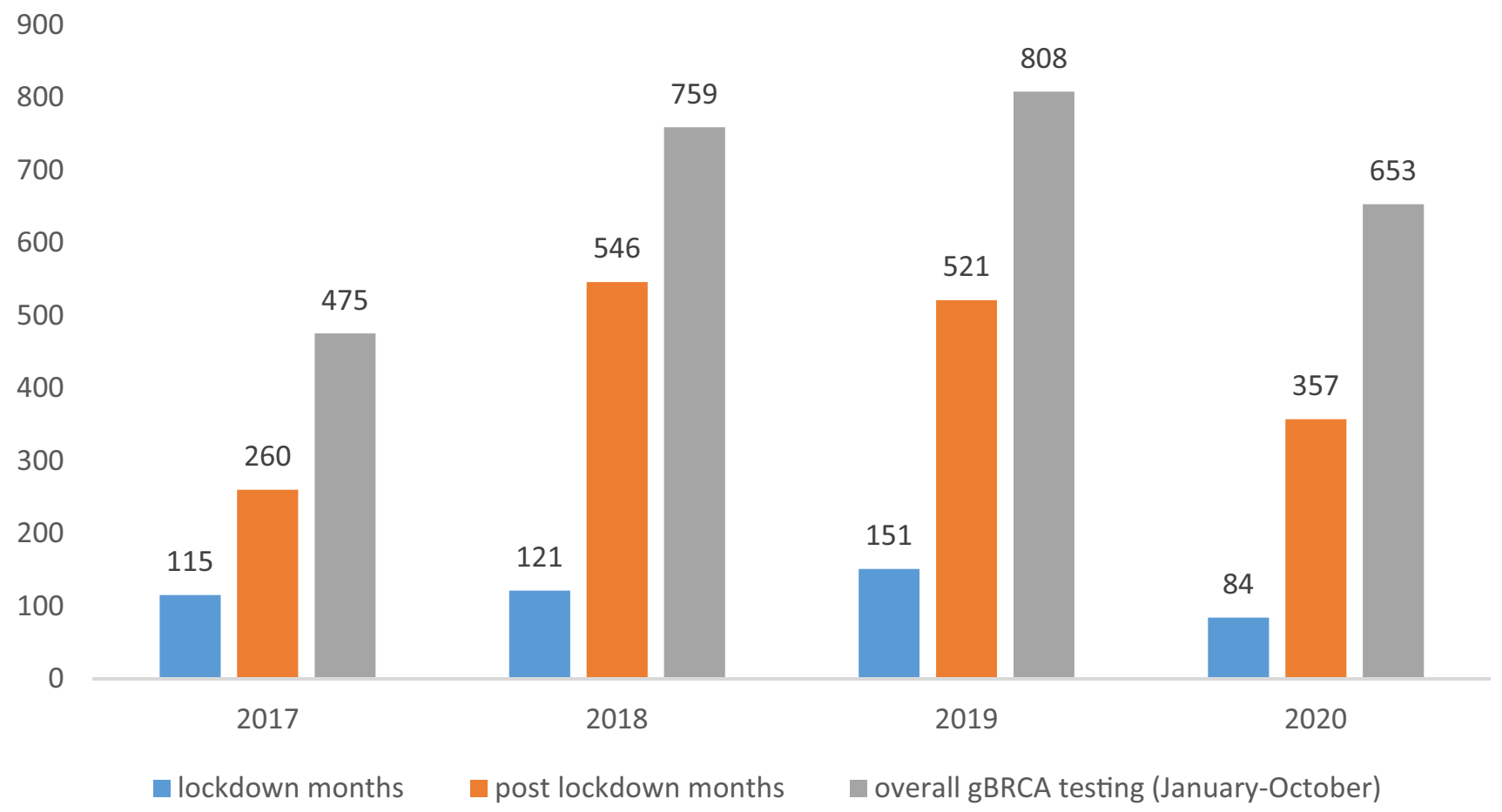

Fig. 2 Germline BRCA tests performed during the lockdown months the tests are significantly lower than in all 3 years considered. Even comparing the trend of the post lockdown months, the tests performed in 2020 are significantly reduced compared to 2019 and 2018
( $-35 \%$ and $-32 \%$, respectively) and are only increased when compared with $2017(+37 \%)$. As consequence, comparing the overall tests/year (Jan-Oct) of the last 3 years, the impact of COVID-19 on $g B R C A$ testing is remarkable 


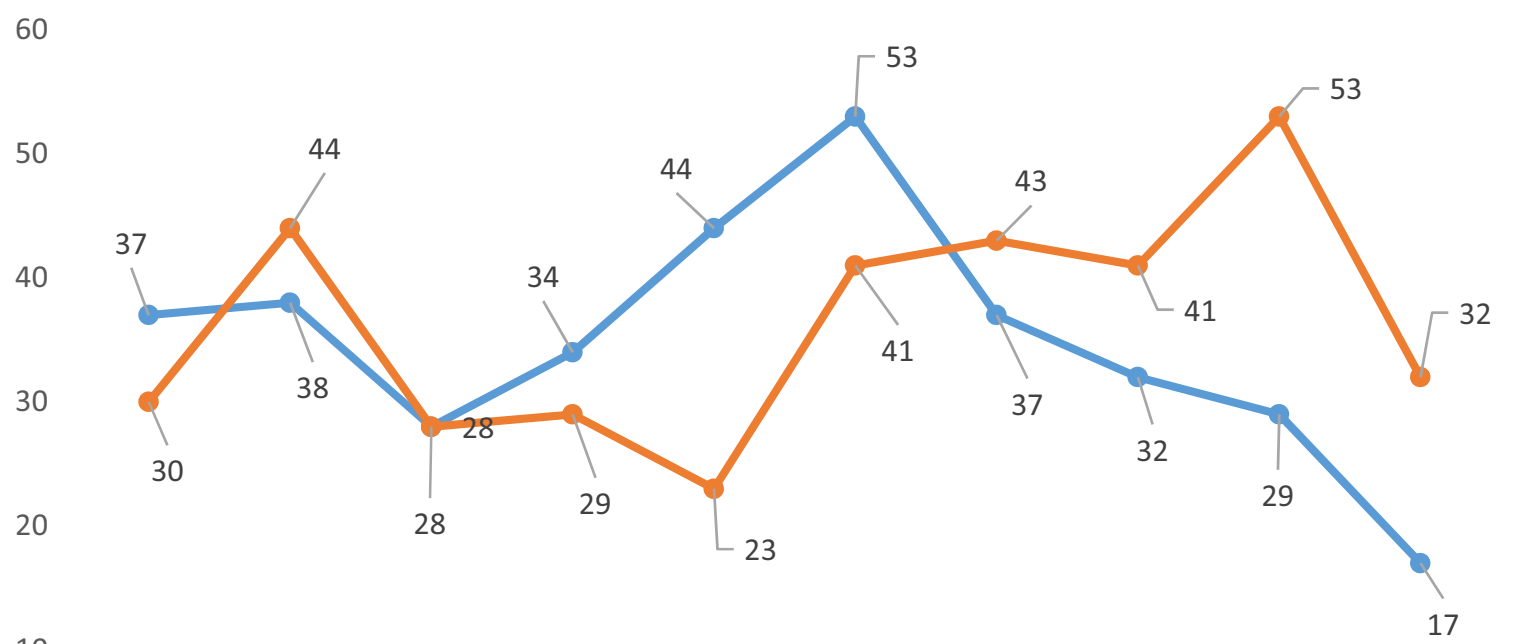

10

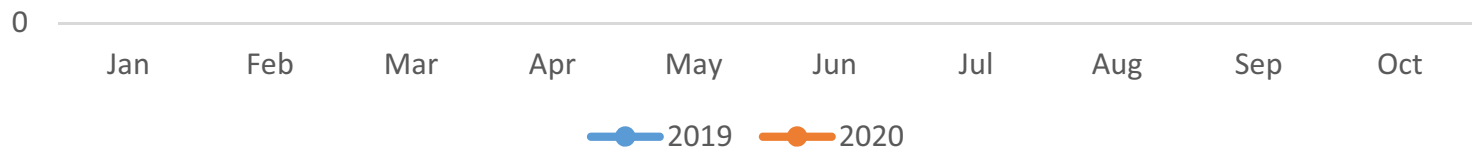

Fig. 3 Impact of COVID-19 pandemic on $t B R C A$ testing from fresh frozen tissue offered in a surgery setting during the 2020 year (January-October). The COVID-19 did not affect the overall $t B R C A$ tests

drastic reduction in requests could be justified by the lockdown imposed by the government, we wanted to confirm our data by analyzing the progress of $g B R C A$ testing requests even after the end of the Italian lockdown. This analysis highlighted that the post lockdown did not cause a significant change in the overall $g B R C A$ testing. Even comparing the overall tests/year (January-October) of the last 3 years, the impact of COVID-19 on $g B R C A$ testing is remarkable.

These evidences indicate presently a $g B R C A$ testing delay for cancer patients and healthy patients. Hereditary $B R C A$ related tumors are syndromes characterized by the development of different types of cancer in succession. Taking advantage of knowing the predisposition of susceptibility for these cancers by $g B R C A$ testing, we underline that it is vital to continue adequate patient management and treatment, including the adoption of preventive measures and countermeasures.

\section{How to act?}

We propose some measures, which could ensure and increase access to $g B R C A$ testing:

I. Promote the message that healthy people and cancer patients should resume routine cancer clinical/diagnostic screenings and not delay care;
(349 $t B R C A$ tests on 2019 versus 364). Tumor BRCA testing had a delay only in the months of lockdown in Italy, as expected

II. Streamline hospital access for patients who undergo molecular tests such as $g B R C A$ testing, where fasting is not necessary;

III. Offer innovative $g B R C A$ testing modalities (e.g., blood sampling at home sending samples to the lab);

IV. Reassure patients safety by wearing protective measures, if they may avoid $g B R C A$ testing due to fears of contracting COVID-19 in hospitals;

V. Offer $B R C A$ testing in obligatory and non-postponable contexts (e.g., surgery, chemotherapy sessions); specifically, BRCA testing offered during surgery could be a winning strategy. Furthermore, it may also be convenient to obtain a blood sample when the tissue sample is collected. In this case, if the tissue test is positive or inconclusive, the blood test could reveal further results. This would reduce access to COVID19 hospitals for cancer patients.

\section{Limitations}

A limitation of this study is that the evidence regarding the execution of $g B R C A$ testing refers to a single center, albeit a reference center for $B R C A$ testing in Italy. We are aware that our analysis would have been significantly strengthened including data of additional genetic centers. 


\section{Conclusions}

Our research provides firsts insights and further studies, involving multiple $B R C A$ referral centers, are required to assess impacts of the pandemic. We emphasize that by applying innovative strategies continuity of oncogenetic tests (e.g., $g B R C A$ testing) can be guaranteed in the presence of this serious ongoing COVID-19 pandemic. Therefore, increased cooperation and multidisciplinary care, changes in procedures and organization, as well as health campaigns, which raise awareness among patients and clinicians, are necessary. This involves a great effort of diverse health care professionals, including public health, and specifically of those involved in the $g B R C A$ testing process.

Acknowledgements We would like to thank Franziska M. Lohmeyer, $\mathrm{PhD}$, for critically reviewing and editing our manuscript.

\section{Compliance with ethical standards}

Conflict of interest The authors declared no potential conflicts of interest.

Ethical approval This study complied with the Ethical Principles for Medical Research Involving Human Subjects according to the World Medical Association Declaration of Helsinki and was certified by the Committee of the Applicable Institution of the Fondazione Policlinico Universitario Agostino Gemelli IRCCS, Rome.

Informed consent Informed consent was taken from all patients who underwent $B R C A$ testing.

\section{References}

1. García-Álvarez L, de la Fuente-Tomás L, García-Portilla MP et al (2020) Early psychological impact of the 2019 coronavirus disease (COVID-19) pandemic and lockdown in a large Spanish sample. J Glob Health 10:020505. https://doi.org/10.7189/ jogh.10.020505

2. Quaquarini E, Saltalamacchia G, Presti D et al (2020) Impact of COVID-19 outbreak on Cancer patient care and treatment: data from an outpatient oncology clinic in Lombardy (Italy). Cancers (Basel) 12:E2941. https://doi.org/10.3390/cancers 12102941

3. Sha Z, Chang K, Mi J et al (2020) The impact of the COVID-19 pandemic on lung cancer patients. Ann Palliat Med 9:3373-3378. https://doi.org/10.21037/apm-20-1662

4. Brunetti O, Derakhshani A, Baradaran B et al (2020) COVID19 infection in Cancer patients: how can oncologists deal with these patients? Front Oncol 10:734. https://doi.org/10.3389/ fonc. 2020.00734

5. Konstantinopoulos PA, Norquist B, Lacchetti C et al (2020) Germline and somatic tumor testing in epithelial ovarian cancer: ASCO guideline. J Clin Oncol 38:1222-1245. https://doi. org/10.1200/JCO.19.02960
6. Hirsch S, Gieldon L, Sutter C et al (2020) Germline testing for homologous recombination repair genes - opportunities and challenges. Genes Chromosomes Cancer 20. https://doi.org/10.1002/ gcc. 22900

7. Moore K, Colombo N, Scambia G et al (2018) Maintenance olaparib in patients with newly diagnosed advanced ovarian cancer. $\mathrm{N}$ Engl J Med 379:2495-2505. https://doi.org/10.1056/NEJMoa1810 858 pmid:http://www.ncbi.nlm.nih.gov/pubmed/30345884

8. Zimmer AS, Gillard M, Lipkowitz S (2018) Update on PARP inhibitors in breast Cancer. Curr Treat Options in Oncol 19:21. https://doi.org/10.1007/s11864-018-0540-2

9. Mateo J, Carreira S, Sandhu S (2015) DNA-repair defects and Olaparib in metastatic prostate Cancer. N Engl J Med 373:16971708. https://doi.org/10.1056/NEJMoa1506859

10. Golan T, Hammel P, Reni M et al (2019) Olaparib as maintenance treatment following first-line platinum-based chemotherapy in patients with a germline BRCA mutation and metastatic pancreatic cancer: phase III POLO trial. Ann Oncol 30(Suppl 4):iv152. https://doi.org/10.1093/annonc/mdz183

11. Bardelli A (2020) Coronavirus lockdown: what I learnt when I shut my cancer lab in 48 hours. Nature 19. https://doi.org/10.1038/ d41586-020-00826-7

12. Malapelle U, Pisapia P, Iaccarino A et al (2020) Predictive molecular pathology in the time of coronavirus disease (COVID-19) in Europe. J Clin Pathol 31:206957. https://doi.org/10.1136/jclin path-2020-206957

13. Minucci A, Scambia G, Santonocito C et al (2015) Clinical impact on ovarian cancer patients of massive parallel sequencing for BRCA mutation detection: the experience at Gemelli hospital and a literature review. Expert Rev Mol Diagn 15:1383-1403. https:// doi.org/10.1586/14737159.2015.1081059

14. Minucci A, De Paolis E, Concolino P et al (2017) Competitive PCR-high resolution melting analysis (C-PCR-HRMA) for large genomic rearrangements (LGRs) detection: a new approach to assess quantitative status of BRCA1 gene in a reference laboratory. Clin Chim Acta 470:83-92. https://doi.org/10.1016/j. cca.2017.04.026

15. Santonocito C, Scapaticci M, Guarino D et al (2017) Identification of twenty-nine novel germline unclassified variants of BRCA1 and BRCA2 genes in 1400 Italian individuals. Breast. 36:74-78. https ://doi.org/10.1016/j.breast.2017.09.007

16. Minucci A, Scambia G, Santonocito C et al (2020) BRCA testing in a genomic diagnostics referral center during the COVID-19 pandemic. Mol Biol Rep 47:4857-4860. https://doi.org/10.1007/ s11033-020-05479-3

17. Marchetti C, Minucci A, D'Indinosante M et al (2020) Feasibility of tumor testing for BRCA status in high-grade serous ovarian cancer using fresh-frozen tissue based approach. Gynecol Oncol 158:740-746. https://doi.org/10.1016/j.ygyno.2020.06.479

18. Sud A, Torr B, Jones ME et al (2020) Effect of delays in the 2-week-wait cancer referral pathway during the COVID-19 pandemic on cancer survival in the UK: a modelling study. Lancet Oncol 21:1035-1044. https://doi.org/10.1016/S1470 $-2045(20) 30392-2$

Publisher's Note Springer Nature remains neutral with regard to jurisdictional claims in published maps and institutional affiliations. 